\title{
Reflexivity of the isometry group of some classical spaces
}

Félix Cabello Sánchez and Lajos Molnár

\begin{abstract}
We investigate the reflexivity of the isometry group and the automorphism group of some important metric linear spaces and algebras. The paper consists of the following sections: 1. Preliminaries. 2. Sequence spaces. 3. Spaces of measurable functions. 4. Hardy spaces. 5. Banach algebras of holomorphic functions. 6. Fréchet algebras of holomorphic functions. 7. Spaces of continuous functions.
\end{abstract}

\section{Introduction.}

This paper is concerned with the reflexivity of the isometry group and the automorphism group of certain particular, but important, topological vector spaces and algebras. Although we deal mainly with Banach spaces, we are also interested in other (not necessarily locally convex) metric linear spaces and some Fréchet algebras.

Reflexivity problems for subalgebras of the algebra of all bounded linear operators acting on a Hilbert space represent one of the most active research areas in operator theory. The study of similar questions concerning sets of linear transformations on Banach algebras rather than on Hilbert spaces was initiated by Kadison [10] and Larson [14]. In [10], motivated by the study of Hochschild cohomology of operator algebras, the reflexivity of the Lie algebra of all derivations on a von Neumann algebra

2000 Mathematics Subject Classification: 47B49, 46B04, 47B10.

Keywords: Reflexivity, Isometry, Automorphism. 
was treated. In [14, Some concluding remarks (5), p. 298], Larson raised the question of the reflexivity of the automorphism group of Banach algebras. This problem was investigated for several algebras in [1], [19], [20], [21] and [23]. The present article is a continuation of that work.

We describe the results of the paper as follows. The first section is preliminary. In Section 2 we investigate symmetric spaces. We prove that, with the sole exception of $l_{2}$, every $F$-space with a symmetric basis has algebraically reflexive isometry group. Curiously enough, the isometry group of any non-separable "symmetric" space fails to be algebraically reflexive. Section 3 concentrates on Lebesgue spaces. We show the extreme nonreflexivity of the isometry group of the spaces $L_{p}(\mu)(0<p<\infty)$ for homogeneous measures $\mu$, thus obtaining that the only infinite dimensional Lebesgue spaces whose isometry groups are reflexive are the sequence spaces $l_{p}$ for $p \neq 2$. In contrast, the isometry groups of the Hardy spaces $H^{p}(0<p<\infty)$ are topologically reflexive for all $p \neq 2$. This will be proved in Section 4.

Sections 5 and 6 deal with algebras of holomorphic functions. We prove that the isometry group and the automorphism group of the disc algebra are topologically reflexive. The same is true for $H^{\infty}(\Omega), \Omega$ being any simply connected domain in the plane. Furthermore, we consider the "unbounded" case: it is shown that the automorphism group of the Fréchet algebra $H(\Omega)$ is topologically reflexive if and only if $\Omega \neq \mathbb{C}$.

Finally, we study algebras of continuous functions. We solve some problems posed in [22] by presenting Banach spaces whose isometry groups are either trivial or very large. We give an example of a compact Hausdorff space $K$ such that the isometry group and the automorphism group of the Banach algebra $C(K)$ both fail to be reflexive, thus verifying a conjecture formulated in [22], where it was proved that the isometry group and the automorphism group of $C(K)$ are algebraically reflexive in case $K$ is first countable. Furthermore, we exhibit a Banach space $X$ with the property that its isometry group is topologically reflexive but the isometry group of its dual space $X^{*}$ is even algebraically nonreflexive. Next, we present another Banach space $Y$ whose isometry group is not algebraically reflexive and yet the isometry group of $Y^{*}$ is algebraically reflexive. 


\section{Preliminaries.}

Let $X$ be a topological vector space and let $B(X)$ be the algebra of all continuous linear operators on $X$. Given any subset $\mathcal{S} \subset B(X)$, define

$$
\begin{aligned}
& \operatorname{ref}_{a l} \mathcal{S}=\{T \in B(X): T x \in \mathcal{S} x \text { for all } x \in X\} \\
& \operatorname{ref}_{t o} \mathcal{S}=\{T \in B(X): T x \in \overline{\mathcal{S} x} \text { for all } x \in X\}
\end{aligned}
$$

where $\mathcal{S} x=\{L x: L \in \mathcal{S}\}$ and the bar stands for the closure in $X$. The set $\mathcal{S}$ is said to be algebraically reflexive if $\operatorname{ref}_{a l} \mathcal{S}=\mathcal{S}$ and, similarly, $\mathcal{S}$ is called topologically reflexive if $\operatorname{ref}_{t o} \mathcal{S}=\mathcal{S}$. Thus, reflexive sets of operators are, in some sense, completely determined by their local actions on the underlying space. Sometimes the operators in $\operatorname{ref}_{a l} \mathcal{S}$ are said to belong locally to $\mathcal{S}$.

Since there is no clear intrinsic reason to restrict our attention to the locally convex setting when dealing with local surjective isometries, we consider $F$-spaces, not only Banach spaces.

Recall from $[12$, p. 2$]$ that a $\Delta$-norm on a real or complex vector space $X$ is a non-negative real-valued function on $X$ satisfying

1) $\|x\|>0$ for all $0 \neq x \in X$.

2) $\|\alpha x\| \leq\|x\|$ for all $x \in X$ and all $\alpha \in \mathbb{K}$ with $|\alpha| \leq 1$.

3) $\lim _{\alpha \rightarrow 0}\|\alpha x\|=0$ for all $x \in X$.

4) $\|x+y\| \leq K(\|x\|+\|y\|)$ for some constant $K$ independent on $x, y \in X$.

A $\Delta$-norm on $X$ induces a metrizable linear topology for which the sets $U_{n}=\{x \in X:\|x\|<1 / n\}$ form a neighbourhood base at the origin and, conversely, every metrizable linear topology comes from a $\Delta$-norm. An $F$-norm is a $\Delta$-norm satisfying

5) $\|x+y\| \leq\|x\|+\|y\|$ for all $x, y \in X$.

Any $F$-norm induces a translation-invariant metric on $X$ in the obvious way and every invariant metric compatible with the linear structure is induced by some $F$-norm. An $F$-space is a complete $F$-normed space.

Finally, a quasi-norm is a $\Delta$-norm which is homogeneous in the sense that

6) $\|\alpha x\|=|\alpha|\|x\|$ for all $x \in X, \alpha \in \mathbb{K}$. 
Observe that 6) implies both 2) and 3) so that quasi-norms can be defined by 1),6) and 4). A quasi-normed space is a vector space together with some specified quasi-norm. Such a space is locally bounded, that is, it has a bounded neighbourhood of zero. Conversely, every locally bounded topology is induced by a quasi-norm. A quasi-Banach space is a complete quasi-normed space.

We denote by Iso $(X)$ the group of all surjective (linear) isometries of the $\Delta$-normed space $X$. Also, when $\mathcal{A}$ is a topological algebra, $\operatorname{Aut}(\mathcal{A})$ denotes the group of all continuous automorphisms of $\mathcal{A}$. In accordance with what is written above, we call the elements of $\operatorname{ref}_{a l}(\operatorname{Iso}(X))$ and $\operatorname{ref}_{a l}(\operatorname{Aut}(\mathcal{A}))$ local surjective isometries and local automorphisms, respectively.

One little problem with $\Delta$-norms is that a $\Delta$-norm need not be continuous with respect to the topology it induces. (The continuity of a norm is a consequence of the triangle inequality.) This has some unpleasant consequences. For instance, the operators in $\operatorname{ref}_{t o}(\operatorname{Iso}(X))$ need not be into isometries. An interesting class of continuous quasi-norms is that of the so-called $p$-norms $(0<p \leq 1)$. These are quasi-norms satisfying

$$
\|x+y\|^{p} \leq\|x\|^{p}+\|y\|^{p}
$$

from which continuity immediately follows. Clearly, if $\|\cdot\|$ is a $p$-norm, then $\|\cdot\|^{p}$ is an $F$-norm with the same isometries.

\section{Sequence spaces.}

In this section we study sequence spaces. A basis of an $F$-space $X$ is a sequence $\left\{e_{n}\right\}$ so that every $x \in X$ has a unique expansion $x=\sum_{n=1}^{\infty} x_{n} e_{n}$. A basis $\left\{e_{n}\right\}$ is said to be symmetric if

$$
\left\|\sum_{n=1}^{\infty} x_{n} e_{n}\right\|=\left\|\sum_{n=1}^{\infty} \varepsilon_{n} x_{n} e_{\pi(n)}\right\|
$$

holds for every choice of scalars $\varepsilon_{n}$ of modulus 1 and every permutation $\pi$ of the positive integers. Perhaps the most interesting class of spaces with a symmetric basis is that of the Orlicz sequence spaces (see [12], [18], [25] for definitions) which contains the Banach spaces $l_{p}$ for $1 \leq p<\infty$, the quasi-Banach spaces $l_{p}$ for $0<p<1$ and other locally bounded and even non-locally bounded spaces. Another important class related to $l_{p}$ spaces is that of the Lorentz sequence spaces [18]. 
Theorem 1. Let $X$ be an $F$-space not isomorphic to $l_{2}$. Suppose that $X$ has a symmetric basis. Then the isometry group of $X$ is algebraically reflexive.

Proof. By a result of Rolewicz [25, Theorems 9.8.3 and 9.8.5] (see also [7]), every surjective isometry of $X$ is of the form

$$
\sum x_{n} e_{n} \longmapsto \sum \varepsilon_{n} x_{n} e_{\pi(n)}
$$

where $\left|\varepsilon_{n}\right|=1$ and $\pi$ is a permutation of $\mathbb{N}$. It follows that if $T$ is a local surjective isometry of $X$, then there is an injective mapping $\varphi$ on $\mathbb{N}$ for which

$$
T e_{n}=\sigma_{n} e_{\varphi(n)},
$$

where $\left|\sigma_{n}\right|=1$. Hence $T$ is given by

$$
T\left(\sum x_{n} e_{n}\right)=\sum \sigma_{n} x_{n} e_{\varphi(n)}
$$

The theorem will be proved if we show that $\varphi$ is surjective. It is easily seen that $X$ contains an $x=\sum x_{n} e_{n}$ with $x_{n}>0, x_{n} \neq x_{m}(n \neq m)$. Taking $L \in \operatorname{Iso}(X)$ so that $T x=L x$, one obtains that

$$
\sum \sigma_{n} x_{n} e_{\varphi(n)}=\sum \varepsilon_{n} x_{n} e_{\pi(n)}
$$

for some permutation $\pi$ of $\mathbb{N}$. Hence $\varphi=\pi, \varphi$ is surjective and the proof is complete.

Remark 1. Observe that, in general, we do not have topological reflexivity in Theorem 1. In fact, let $X=c_{0}$ and consider the unilateral shift $S$ on $X$. It is easy to see that for every $x \in X$ and $\varepsilon>0$ there exists a surjective isometry $L$ such that $\|S x-L x\|<\varepsilon$. Therefore, $S \in \operatorname{ref}_{t o}(\operatorname{Iso}(X))$ but $S$ is not surjective.

The following example shows that separability is essential in Theorem 1.

Example 1. Let $X$ be a $\Delta$-normed space of functions on an index set $\Gamma$. Suppose that

a) for every $f \in X$ the set $\{\gamma \in \Gamma: f(\gamma) \neq 0\}$ is at most countable,

b) for every bijection $\varphi$ of $\Gamma$, the map $f \longmapsto f \circ \varphi$ is a surjective isometry of $X$. 
If $\Gamma$ is uncountable, then the isometry group of $X$ is algebraically nonreflexive.

Proof. We closely follow [23, last Remark]. Let $\Lambda$ be a proper subset of $\Gamma$ with a bijection $\varphi: \Lambda \longrightarrow \Gamma$. Define $T: X \longrightarrow X$ by

$$
(T f)(\gamma)= \begin{cases}f(\varphi(\gamma)), & \text { if } \gamma \text { belongs to } \Lambda \\ 0, & \text { elsewhere }\end{cases}
$$

Obviously, $T$ is non-surjective. To see that $T \in \operatorname{ref}_{a l}(\operatorname{Iso}(X))$, fix $f \in X$. Since $f$ has at most countable support one can find a bijection $\phi: \Gamma$ $\varphi^{-1}(\operatorname{supp}(f)) \longrightarrow \Gamma \backslash \operatorname{supp}(f)$. Define a bijection on $\Gamma$ by

$$
\varphi_{f}(\gamma)= \begin{cases}\varphi(\gamma), & \text { if } \gamma \in \varphi^{-1}(\operatorname{supp}(f)) \\ \phi(\gamma), & \text { elsewhere }\end{cases}
$$

Let $T_{f}$ be given on $X$ by $T_{f}(g)=g \circ \varphi_{f}$. Then $T_{f} \in \operatorname{Iso}(X)$ and $T_{f}(f)=$ $T(f)$. This completes the proof.

Remark 2. It is well-known that the isometry group of any infinite dimensional real or complex Hilbert space fails to be algebraically reflexive. This is because Hilbert spaces are isotropic: given $x, y \in H$ with $\|x\|=\|y\|$ there is $T \in \operatorname{Iso}(H)$ such that $y=T x$. Hence $\operatorname{ref}_{a l}(\operatorname{Iso}(H))$ is as large as possible and contains all into isometries. Therefore, Iso $(H)$ cannot be reflexive unless $H$ is finite dimensional. In fact, the isometry group of any infinite dimensional complex Hilbert space is algebraically nonreflexive not only with respect to the original Hilbert space norm, but also with respect to the so-called spin norms [22, Theorem 3.7]. It is natural to ask if the same occurs with any equivalent norm (or renorming, in short). Although in [22] an affirmative answer was conjectured, the following result shows that the answer is strongly negative.

Theorem 2. Every Banach space admits a renorming whose isometry group is topologically reflexive.

Proof. Let $Y$ be a Banach space. By a result of Jarosz [9], there is a renorming $X$ of $Y$ with trivial isometries, that is, $\operatorname{such}$ that $\operatorname{Iso}(X)=$ $\{\sigma I: \sigma \in \mathbb{K},|\sigma|=1\}$. Clearly, Iso $(X)$ is topologically reflexive.

Remark 3. Suppose that $Y$ is a Hilbert space. Using [27] instead of [14] one obtains that for every $\varepsilon>0$ there is a Banach space $X$ with trivial 
isometries and $\varepsilon$-isometric to $Y$ in the sense that there is an isomorphism $T: X \longrightarrow Y$ with $\|T\| \cdot\left\|T^{-1}\right\|<1+\varepsilon$. This means that the new norm can be chosen to be a very small perturbation of the original Hilbert space norm of $Y$.

\section{Spaces of measurable functions.}

Let $(\Omega, \Sigma, \mu)$ be a measure space. For $0<p<\infty$, define $L_{p}(\mu)$ to be the space of all real or complex measurable functions on $\Omega$ for which

$$
\|f\|_{p}=\left(\int_{\Omega}|f|^{p} d \mu\right)^{1 / p}
$$

is finite with the usual convention about identifying functions equal almost everywhere. Observe that $\|\cdot\|_{p}$ is a norm only if $p \geq 1$. For $0<p<1$ it is only a quasi-norm (in fact, a $p$-norm) and $L_{p}(\mu)$ is a quasi-Banach space.

By a famous theorem of Maharam, every Lebesgue space (that is, $L_{p}$ space) is isometrically representable as

$$
L_{p}(\mu)=l_{p}(\Gamma) \oplus_{p}\left(\sum_{i \in I} L_{p}\left(\lambda^{\mathfrak{c}_{i}}\right)\right)_{p},
$$

where $\Gamma$ and $I$ are (possibly empty) sets, $\mathfrak{c}_{i}$ are infinite cardinals and $\lambda$ denotes the Lebesgue measure on $[0,1]$ (observe that, for instance, $L_{p}(\lambda)=$ $\left.L_{p}\left(\lambda^{\omega}\right)\right)$. The subscript $p$ indicates that the corresponding direct sum is taken in the $l_{p}$ sense.

Clearly, $\mu$ is $\sigma$-finite if and only if $\Gamma$ and $I$ are countable sets. Also, $\mu$ is homogeneous in the sense of [8] if and only if $\Gamma$ is empty and all $\mathfrak{c}_{i}$ coincide (apart from the trivial case when $I$ is empty and $\Gamma$ is a singleton). Homogenity means that $\left.\Sigma\right|_{\Omega^{\prime}}$ and $\left.\Sigma\right|_{\Omega^{\prime \prime}}$ are Boolean isomorphic whenever $\Omega^{\prime}$ and $\Omega^{\prime \prime}$ are subsets of $\Omega$ with positive finite measure, which implies that, for every $0<p<\infty$, the spaces $L_{p}\left(\Omega^{\prime}, \mu\right)$ and $L_{p}\left(\Omega^{\prime \prime}, \mu\right)$ are isometrically isomorphic. In that case the isometry group of $L_{p}(\mu)$ has at most two orbits on the unit sphere. More precisely, given $f, g \in L_{p}(\mu)$ with $\|f\|_{p}=$ $\|g\|_{p} \neq 0$, there is a surjective isometry of $L_{p}(\mu)$ mapping $f$ into $g$ if and only if either both $f$ and $g$ are nonzero almost everywhere or both $f$ and $g$ vanish on sets of positive measure (which follows from [8, Lemma 1.4]). This obviously implies that these spaces are almost isotropic: given $f, g \in$ $L_{p}(\mu)$ with $\|f\|_{p}=\|g\|_{p}=1$ and $\varepsilon>0$ there is $T \in \operatorname{Iso}\left(L_{p}(\mu)\right)$ fulfilling $\|g-T f\|_{p} \leq \varepsilon$. If, in addition to be homogeneous, $\mu$ is not $\sigma$-finite, then $L_{p}(\mu)$ is isotropic for all $0<p<\infty$. 
Theorem 3. Let $X$ be an infinite dimensional Lebesgue space. Then $\operatorname{Iso}(X)$ is algebraically reflexive if and only if $X=l_{p}(\mathbb{N})$ with $p \neq 2$.

Proof. The standard basis of $l_{p}(\mathbb{N})$ is symmetric for every $0<p<\infty$, so Theorem 1 implies that $\operatorname{Iso}\left(l_{p}(\mathbb{N})\right)$ is algebraically reflexive for every $p \neq 2$.

For the converse we need the following lemma whose easy proof is left to the reader.

Lemma 1. Let $X$ be a $\Delta$-normed space whose isometry group is algebraically reflexive and let $Y$ be a linear subspace of $X$. Suppose that $Y$ has a complement $Z$ in $X$ such that $\|y+z\|=\Phi(\|y\|,\|z\|)$ holds for some function $\Phi: \mathbb{R}^{2} \longrightarrow \mathbb{R}$ and all $y \in Y, z \in Z$. Then $\operatorname{Iso}(Y)$ is algebraically reflexive too.

Now, it clearly suffices to see that $\operatorname{Iso}(X)$ is algebraically nonreflexive for $X$ equal either $L_{p}\left(\lambda^{\mathfrak{c}}\right)$ or $l_{p}(\Gamma)$ with $\Gamma$ uncountable and then apply Lemma 1. But $l_{p}(\Gamma)$ is just a particular case of Example 1. The following result ends the proof of Theorem 3 .

Lemma 2. For any cardinal $\mathfrak{c}$ the isometry group of the space $L_{p}\left(\lambda^{\mathfrak{c}}\right)$ is algebraically nonreflexive.

Proof. First observe that two functions $f$ and $g$ in an arbitrary $L_{p}$ space (with $p \neq 2$ ) have almost disjoint supports (that is, $f g=0$ holds almost everywhere) if and only if

$$
\|f+g\|_{p}^{p}+\|f-g\|_{p}^{p}=2\left(\|f\|_{p}^{p}+\|g\|_{p}^{p}\right) .
$$

It follows that if $T$ is an into isometry between $L_{p}$ spaces and $f$ vanishes on a set of positive measure, then so does $T f$. (Hence, if in addition $T$ is surjective, then $T f$ is nonzero almost everywhere if and only if $f$ is nonzero almost everywhere.)

Thus, in view of the structure of the orbits of the isometry group of $L_{p}(\mu)$ for $\mu$ a homogeneous measure, it is clear that an into isometry of $L_{p}\left(\lambda^{\mathfrak{c}}\right)$ is locally surjective if and only if it preserves the almost everywhere nonzero functions as well as the functions with support of positive measure.

Let $\Omega=[0,1]^{\mathfrak{c}}$. Write $\Omega=\Omega^{\prime} \oplus \Omega^{\prime \prime}$ with $\Omega^{\prime}, \Omega^{\prime \prime} \in \Sigma$ of positive measure. Obviously, $L_{p}\left(\Omega, \lambda^{\mathfrak{c}}\right)=L_{p}\left(\Omega^{\prime}, \lambda^{\mathfrak{c}}\right) \oplus_{p} L_{p}\left(\Omega^{\prime \prime}, \lambda^{\mathfrak{c}}\right)$. By the homogenity of $\lambda^{\mathfrak{c}}$, there are surjective isometries $T^{\prime}: L_{p}\left(\Omega, \lambda^{\mathfrak{c}}\right) \longrightarrow L_{p}\left(\Omega^{\prime}, \lambda^{\mathfrak{c}}\right)$ and $T^{\prime \prime}: L_{p}\left(\Omega, \lambda^{\mathfrak{c}}\right) \longrightarrow L_{p}\left(\Omega^{\prime \prime}, \lambda^{\mathfrak{c}}\right)$. Define $T: L_{p}\left(\lambda^{\mathfrak{c}}\right) \longrightarrow L_{p}\left(\lambda^{\mathfrak{c}}\right)$ as

$$
T(f)=\frac{T^{\prime}(f)+T^{\prime \prime}(f)}{2^{1 / p}} .
$$


Clearly, $T$ is a non-surjective into isometry. On the other hand, it is easily seen that $T f$ is nonzero almost everywhere if and only if $f$ is. Therefore, $T$ is a local surjective isometry of $L_{p}\left(\Omega, \lambda^{\mathfrak{c}}\right)$. This proves the lemma.

Remark 4. Let $\varphi$ be an Orlicz function (see [12, p. 29]). Then $L_{\varphi}(\mu)$, the Orlicz function space determined by $\varphi$ on the finite measure space $(\Omega, \Sigma, \mu)$ is the space of all measurable functions on $\Omega$ for which

$$
\|f\|_{\varphi}=\int_{\Omega} \varphi(|f(\omega)|) d \mu
$$

is finite. It can be proved that $\|\cdot\|_{\varphi}$ is a complete $\Delta$-norm on $L_{\varphi}(\mu)$. Under some additional hypotheses (e.g. concavity) $\|\cdot\|_{\varphi}$ is even an $F$ norm. It would be interesting to know whether the isometry group of the spaces $L_{\varphi}(\mu)$ is reflexive. For instance, we do not known whether the isometry group of the space of all measurable functions on $[0,1]$ is algebraically reflexive (that space is determined by the concave Orlicz function $\varphi(t)=t /(1+t))$. It should be noted that, with the exception of the Lebesgue spaces $L_{p}(\mu)$ (which correspond to the Orlicz functions $t \mapsto t^{p}$ ), every surjective isometry on a "reasonable" $L_{\varphi}(\mu)$ is induced by a measurepreserving automorphism of $\Sigma$ (see [13]).

\section{Hardy spaces.}

In the sequel, we denote by $U$ the open unit disc in the plane and by $\mathbb{T}$ the unit circle. For $0<p<\infty$, the Hardy space $H^{p}$ is the space of all holomorphic functions $f: U \longrightarrow \mathbb{C}$ for which

$$
\|f\|_{p}=\sup _{0 \leq r<1}\left(\frac{1}{2 \pi} \int_{0}^{2 \pi}\left|f\left(r e^{i \theta}\right)\right|^{p} d \theta\right)^{1 / p}
$$

is finite (the space $H^{\infty}$ will be treated in the next section). If $p \geq 1$, then $\|\cdot\|_{p}$ is a complete norm on $H^{p}$, while for $0<p<1$ it is only a $p$-norm and $H^{p}$ is a quasi-Banach space.

We refer the reader to [5] for general information about Hardy spaces. Here we only recall the well-known inequality

$$
\left|f\left(r e^{i \theta}\right)\right| \leq 2^{1 / p}(1-r)^{-p}\|f\|_{p}, \quad r<1, f \in H^{p},
$$

(see [5, p. 36, Lemma] which implies that for every $z \in U$, the point evaluation $f \longmapsto f(z)$ is continuous on $H^{p}$. 
Theorem 4. For every $p \neq 2$ the isometry group of $H^{p}$ is topologically reflexive.

Before going into the proof, recall from [6] that every into isometry of $H^{p}(p \neq 2)$ has the form

$$
T f=F \cdot(f \circ \varphi), \quad f \in H^{p},
$$

where $\varphi$ is a non-constant inner function (that is, $|\varphi(z)| \leq 1$ for $|z| \leq 1$ and $|\varphi(z)|=1$ for $|z|=1$ ) and $F \in H^{p}$ (we do not use any connection between $\varphi$ and $F$, but see [6, Theorem 1]). Moreover, $T \in \operatorname{Iso}\left(H^{p}\right)$ if and only if

$$
T f=b\left(\frac{d \varphi}{d z}\right)^{1 / p}(f \circ \varphi), \quad f \in H^{p}
$$

where $b \in \mathbb{T}$ and $\varphi$ is a conformal map of the disc onto itself.

Proof. Let $T \in \operatorname{ref}_{t o}\left(\operatorname{Iso}\left(H^{p}\right)\right)$. Since $T$ is an into isometry, there exists $F$ and $\varphi$ such that $T$ is of the form (1) above. Observe that $F=T(1)$ and $F \varphi=T\left(\right.$ id) (here id denotes the identity function on $U$ ). Take $T_{n}, L_{n} \in$ Iso $\left(H^{p}\right)$ such that $F=\lim _{n} T_{n}(1)$ and $F \varphi=\lim _{n} L_{n}($ id). Taking into account the form of the conformal maps of $U$ onto itself, we see that

$$
\begin{gathered}
F=\lim _{n \rightarrow \infty} b_{n}\left(\frac{1-\left|a_{n}\right|^{2}}{\left(1-\bar{a}_{n} \mathrm{id}\right)^{2}}\right)^{1 / p} \\
F \varphi=\lim _{n \rightarrow \infty} \beta_{n}\left(\frac{1-\left|\alpha_{n}\right|^{2}}{\left(1-\bar{\alpha}_{n} \mathrm{id}\right)^{2}}\right)^{1 / p}\left(\frac{\mathrm{id}-\alpha_{n}}{1-\bar{\alpha}_{n} \mathrm{id}}\right),
\end{gathered}
$$

where $b_{n}, \beta_{n} \in \mathbb{T}$ and $a_{n}, \alpha_{n} \in U$ for all $n$. Without loss of generality one can assume that the sequences $\left\{b_{n}\right\},\left\{\beta_{n}\right\},\left\{a_{n}\right\}$ and $\left\{\alpha_{n}\right\}$ are convergent. Put

$$
b=\lim _{n \rightarrow \infty} b_{n}, \quad \beta=\lim _{n \rightarrow \infty} \beta_{n}, \quad a=\lim _{n \rightarrow \infty} a_{n}, \quad \alpha=\lim _{n \rightarrow \infty} \alpha_{n} .
$$

Clearly, $|b|=|\beta|=1$. We show that $a, \alpha \in U$. Suppose that $|a|=1$. Then, using the continuity of point-evaluations, we have

$$
F(z)=\lim _{n \rightarrow \infty} b_{n}\left(\frac{1-\left|a_{n}\right|^{2}}{\left(1-\bar{a}_{n} z\right)^{2}}\right)^{1 / p}=0
$$


for every $z \in U$. But this implies that $T=0$, a contradiction. Therefore $a \in U$. The fact that $\alpha \in U$ can be proved in a similar way: if we assume that $|\alpha|=1$, then $F \varphi=0$ which means that $T$ is not injective. Consequently, we have $\alpha \in U$. Now, it is easily seen that

$$
\begin{gathered}
F(z)=b\left(\frac{1-|a|^{2}}{(1-\bar{a} z)^{2}}\right)^{1 / p} \\
(F \varphi)(z)=\beta\left(\frac{1-|\alpha|^{2}}{(1-\bar{\alpha} z)^{2}}\right)^{1 / p}\left(\frac{z-\alpha}{1-\bar{\alpha} z}\right) .
\end{gathered}
$$

It remains to show that $a=\alpha$. Indeed, in this case we obtain that $\varphi$ is a conformal map of $U$ onto itself, and that $T$ is of the form (2) which gives us that $T$ is a surjective isometry.

Since $\varphi$ is an inner function on the disc, having in mind the form of $F$ and $F \varphi$, we infer that the expression

$$
\frac{\beta}{b} \frac{z-\alpha}{1-\bar{\alpha} z}\left(\frac{1-|\alpha|^{2}}{1-|a|^{2}}\right)^{1 / p}\left(\frac{(1-\bar{a} z)^{2}}{(1-\bar{\alpha} z)^{2}}\right)^{1 / p}
$$

is of modulus 1 whenever $|z|=1$. It is now clear that the Möbius function

$$
\omega(z)=\sqrt{\frac{1-|\alpha|^{2}}{1-|a|^{2}}}\left(\frac{1-\bar{a} z}{1-\bar{\alpha} z}\right)
$$

leaves $\mathbb{T}$ invariant. It is well-known that any holomorphic function on $U$ which is continuous on $\bar{U}$ and leaves $\mathbb{T}$ invariant is either constant or has at least one zero in $U$. Since in case $a \neq \alpha$ the only zero of $\omega$ is at $1 / \bar{a}$ which is of modulus greater than 1 , it follows that $\omega$ is constant and hence we find that $a=\alpha$. The proof is now complete.

\section{Banach algebras of holomorphic functions.}

In this section we study the isometry group and the automorphism group of Banach algebras of holomorphic functions. If $\Omega \subset \mathbb{C}$ is a domain (that is, an open, connected set), then $H(\Omega)$ denotes the algebra of all holomorphic functions on $\Omega$ and $H^{\infty}(\Omega)$ stands for the algebra of all bounded functions in $H(\Omega)$. If $K \subset \mathbb{C}$ is a compact set, then $A(K)$ denotes the algebra of all continuous functions which are holomorphic in the interior of $K$. On $H(\Omega)$ we consider the topology of the uniform convergence on 
compact subsets, while $H^{\infty}(\Omega)$ and $A(K)$ are equipped with the sup-norm topology.

Let $\mathcal{A}$ be a unital semisimple commutative Banach algebra. $\mathcal{A}$ is called a uniform algebra if the spectral radius $r(\cdot)$ is a complete norm on $\mathcal{A}$. By the well-known fact on the uniqueness of Banach algebra norms on semisimple Banach algebras [24, 6.1.1 Theorem], using the result that an injective linear map between Banach spaces has closed range if and only if it is bounded from below, it is easy to see that $\mathcal{A}$ is a uniform algebra if and only if the range of the Gelfand transformation on $\mathcal{A}$ is a closed subalgebra of the space of all continuous complex valued functions on its structure space (see the definition of sup-norm algebras in [15]). In what follows $\sigma(\cdot)$ denotes the spectrum.

Theorem 5. Let $\mathcal{A}$ be a uniform algebra. Every $T \in \operatorname{ref}_{\text {to }}(\operatorname{Iso}(\mathcal{A}))$ has the form

$$
T(f)=\tau \psi(f), \quad f \in \mathcal{A},
$$

for some $\tau \in \mathcal{A}$ with $\sigma(\tau) \subset \mathbb{T}$ and some unital algebra endomorphism $\psi: \mathcal{A} \longrightarrow \mathcal{A}$.

For the proof we recall the result [15, Theorem 3] stating that every surjective linear isometry of a uniform algebra $\mathcal{A}$ is an algebra automorphism of $\mathcal{A}$ multiplied by an element of $\mathcal{A}$ whose spectrum is contained in T.

Proof. Let $T \in \operatorname{ref}_{t o}(\operatorname{Iso}(\mathcal{A}))$. Denote $\tau=T(1)$. Since

$$
\sigma(\tau)=\{\varphi(\tau): \varphi \text { is a character of } \mathcal{A}\},
$$

and the characters of $\mathcal{A}$ are continuous, we obtain $\sigma(\tau) \subset \mathbb{T}$. Consider the mapping $\psi(\cdot)=\tau^{-1} T(\cdot)$. Let $\varphi$ be an arbitrary character of $\mathcal{A}$. We have $(\varphi \circ \psi)(1)=1$. Let $f \in \mathcal{A}$ be an arbitrary invertible element and choose $\tau_{n} \in \mathcal{A}$ with $\sigma\left(\tau_{n}\right) \subset \mathbb{T}$ and a sequence $\left\{\psi_{n}\right\}$ of automorphisms of $\mathcal{A}$ such that

$$
\psi(f)=\lim _{n \rightarrow \infty} \tau^{-1} \tau_{n} \psi_{n}(f) .
$$

We have

$$
\varphi(\psi(f))=\lim _{n \rightarrow \infty} \varphi(\tau)^{-1} \varphi\left(\tau_{n}\right) \varphi\left(\psi_{n}(f)\right) .
$$

Clearly, the number $\varphi(\tau)^{-1} \varphi\left(\tau_{n}\right)$ is of modulus 1 and $\varphi\left(\psi_{n}(f)\right) \in \sigma(f)$. Since $0 \notin \sigma(f)$ and the spectrum in a unital Banach algebra is compact, it 
follows that the limit in (3) is nonzero. Therefore, the linear functional $\varphi \circ \psi$ maps 1 to 1 and it sends invertible elements to nonzero complex numbers. By the well-known Gleason-Kahane-Żelazko theorem [24, 2.4.13 Theorem] these imply that $\varphi \circ \psi$ is a multiplicative linear functional for every character $\varphi$ of $\mathcal{A}$. From the semisimplicity of $\mathcal{A}$ it follows that $\psi$ is an algebra homomorphism.

Remark 5. In view of the above proof it is obvious that if $\mathcal{A}$ is a (unital) semisimple commutative Banach algebra, then every element of $\operatorname{ref}_{\text {to }}(\operatorname{Aut}(\mathcal{A}))$ is a (unital) algebra endomorphism of $\mathcal{A}$.

Theorem 6. The isometry group and the automorphism group of the disc algebra $A(\bar{U})$ are topologically reflexive.

Before the proof we recall some basic facts about the Banach algebra $A(\bar{U})$. First of all, the structure space of $A(\bar{U})$ is $\bar{U}[24,3.2 .13]$. This gives us that $r(f)=\|f\|$ for every $f \in A(\bar{U})$ which shows that the disc algebra is a uniform algebra. Afterwards, one can easily verify that the automorphisms of $A(\bar{U})$ are precisely the maps of the form

$$
f \longmapsto f \circ \varphi,
$$

where $\varphi: \bar{U} \longrightarrow \bar{U}$ is a homeomorphism which maps $U$ conformally onto itself.

Proof. Let $T \in \operatorname{ref}_{t o}(\operatorname{Iso}(A(\bar{U})))$. By Theorem 5, T is a unital endomorphism multiplied by a function $\tau \in A(\bar{U})$ whose spectrum is contained in $\mathbb{T}$. Since the spectrum of $\tau$ is $\tau(\bar{U})$, we find that $\tau$ must be a constant function of modulus 1 and without loss of generality we can assume that $T$ is actually a unital endomorphism of the disc algebra. It is not hard to verify that $T$ is necessarily of the form

$$
T(f)=f \circ \varphi, \quad f \in A(\bar{U}),
$$

for some function $\varphi: \bar{U} \longrightarrow \bar{U}$. Since

$$
T(\mathrm{id}) \in \overline{\{\lambda \psi(\mathrm{id}):|\lambda|=1, \psi \text { is an automorphism of the disc algebra }\}},
$$

taking into account the form of automorphisms of $A(\bar{U})$ and that of the conformal maps of $U$ onto itself, it follows that there are sequences $\lambda_{n} \in \mathbb{T}$ and $\alpha_{n} \in U$ such that

$$
\varphi(z)=T(\mathrm{id})(z)=\lim _{n \rightarrow \infty} \lambda_{n} \frac{z-\alpha_{n}}{1-\overline{\alpha_{n}} z},
$$


where the convergence is uniform in $z \in \bar{U}$. Choosing subsequences if necessary, we may assume that $\left\{\lambda_{n}\right\}$ and $\left\{\alpha_{n}\right\}$ converge to $\lambda$ and $\alpha$, respectively. Suppose that $\alpha$ is of modulus 1 . Then

$$
\lim _{n \rightarrow \infty} \frac{z-\alpha_{n}}{1-\overline{\alpha_{n}} z}=-\alpha, \quad|z|<1 .
$$

Since the above convergence is uniform, there is an $n \in \mathbb{N}$ such that

$$
\left|\frac{z-\alpha_{n}}{1-\overline{\alpha_{n}} z}+\alpha\right|<\frac{1}{2}, \quad|z|<1
$$

As the function $z \longmapsto\left(z-\alpha_{n}\right) /\left(1-\overline{\alpha_{n}} z\right)$ maps $U$ onto itself, we arrive at a contradiction. Consequently, $\varphi$ is of the form

$$
\varphi(z)=\lambda \frac{z-\alpha}{1-\bar{\alpha} z}, \quad z \in \bar{U}
$$

with $|\lambda|=1$ and $|\alpha|<1$, from which we obtain the surjectivity of $T$. This completes the proof of the topological reflexivity of $\operatorname{Iso}(A(\bar{U}))$.

The statement about $\operatorname{Aut}(A(\bar{U}))$ follows from the same argument.

Theorem 7. Let $\Omega \subset \mathbb{C}$ be a simply connected domain. The isometry group and the automorphism group of $H^{\infty}(\Omega)$ are topologically reflexive.

Proof. Clearly, the spectrum of any element $f$ of $H^{\infty}(\Omega)$ is $\overline{f(\Omega)}$. Therefore, the spectral radius is equal to the norm and hence $H^{\infty}(\Omega)$ is a uniform algebra.

If $\Omega=\mathbb{C}$, then by Liouville's theorem we have $H^{\infty}(\Omega)=\mathbb{C}$ and in this case the statement is trivial.

Suppose that $\Omega \subsetneq \mathbb{C}$. By the Riemann mapping theorem we may assume that $\Omega=U$. First observe that since the structure space of $H^{\infty}(U)$ contains $U$, it follows that every element of $H^{\infty}(U)$ with spectrum contained in $\mathbb{T}$ is a constant function of modulus 1 . Also, we know that every automorphism of the algebra $H^{\infty}(U)$ is induced by a conformal selfmap of $U$ [11]. Now, let $T \in \operatorname{ref}_{t o}\left(\operatorname{Iso}\left(H^{\infty}(U)\right)\right)$. Just as in the proof of Theorem 6 we can assume that $T(1)=1$. One can check in a way very similar to that we have followed there that $T$ (id) is a conformal map of $U$ onto itself. Therefore, we can suppose that even $T$ (id) $=$ id holds true. With all these assumptions assume that $z_{0} \in U$ and that $f \in H^{\infty}(U)$ is such that $f\left(z_{0}\right)=0$. We obtain

$$
\frac{f}{\mathrm{id}-z_{0}} \in H^{\infty}(U)
$$


Since $T$ is a unital algebra homomorphism, it follows that

$$
\frac{T(f)}{\mathrm{id}-z_{0}}=T\left(\frac{f}{\mathrm{id}-z_{0}}\right) \in H^{\infty}(U)
$$

which gives us that $T(f)\left(z_{0}\right)=0$. Let now $f \in H^{\infty}(U)$ be arbitrary. Since the function $f-f\left(z_{0}\right)$ vanishes at $z_{0}$, it follows that the same holds for $T\left(f-f\left(z_{0}\right)\right)=T(f)-f\left(z_{0}\right)$. Thus, we have $T(f)\left(z_{0}\right)=f\left(z_{0}\right)$. Since this is true for every $z_{0} \in U$ and $f \in H^{\infty}(U)$, we have $T(f)=f$ for all $f \in H^{\infty}(U)$. This completes the proof of the theorem.

Remark 6. The main difference between the proofs of Theorem 6 and Theorem 7 is that in the former one we were lucky to use the form of endomorphisms of $A(\bar{U})$. Such an "inner" form for the endomorphisms of $H^{\infty}(U)$ does not exist. The reason is that the structure space of $H^{\infty}(U)$ is much bigger than $U$.

Remark 7. We have some remarks concerning the algebraic reflexivity of the isometry group and the automorphism group of algebras of holomorphic functions on more general domains.

First recall that if $K \subset \mathbb{C}$ is a compact set whose complement has finitely many components, then the structure space of $A(K)$ is just $K$. In fact, this follows from the celebrated Mergelyan's approximation theorem which states that in this case $A(K)$ is equal to the sup-norm closure of the set of all rational functions with poles outside $K[26,20.5$ Theorem and Exercise 1, p. 427], [24, 3.2.14 Example]. We easily obtain that $A(K)$ is a uniform algebra and having a look at the proofs of Theorem 5 and 6 , one can easily verify that the isometry group and the automorphism group of $A(K)$ are algebraically reflexive.

Next, let $\Omega \subset \mathbb{C}$ be a bounded domain. We assert that the isometry group and the automorphism group of $H^{\infty}(\Omega)$ are algebraically reflexive. As for the proof, we can clearly assume that

$$
\Omega \subset\{z \in \mathbb{C}: \operatorname{Re} z>0\}
$$

and that $\Omega$ contains a real number. Obviously, if $\lambda \in \mathbb{T}$ is any number different from 1 , then there is a positive integer $n$ such that $\lambda^{n} \bar{\Omega} \nsubseteq \bar{\Omega}$. After this short preparation, let $T: H^{\infty}(\Omega) \longrightarrow H^{\infty}(\Omega)$ be a local surjective isometry. Just as in the proof of Theorem 7 , we can suppose that $T(1)=1$. Let $\lambda$ be a complex number of modulus 1 and let $\psi$ be an automorphism of $H^{\infty}(\Omega)$ such that

$$
T(\mathrm{id})=\lambda \psi(\mathrm{id})
$$


We note that it is not true for general domains that every automorphism of $H^{\infty}(\Omega)$ is induced by a conformal selfmap of $\Omega$. We show that in (4) we have $\lambda=1$. The spectrum of id is $\bar{\Omega}$. Clearly, every automorphism preserves the spectrum while any unital algebra homomorphism is spectrum non-increasing. Hence, from the relation above we infer that

$$
\lambda \bar{\Omega}=\lambda \sigma(\mathrm{id})=\lambda \sigma(\psi(\mathrm{id}))=\sigma(T(\mathrm{id})) \subset \sigma(\mathrm{id})=\bar{\Omega} .
$$

Since this implies that $\lambda^{n} \bar{\Omega} \subset \bar{\Omega}$ for every $n \in \mathbb{N}$, we obtain that $\lambda$ must equal 1. As the norm in $H^{\infty}(\Omega)$ is equal to the spectral radius, it follows that every automorphism is a surjective isometry. Therefore, considering the map $\psi^{-1} \circ T$ we can assume that our local surjective isometry satisfies $T(1)=1$ and $T(\mathrm{id})=\mathrm{id}$. Now, the proof can be completed as in the last part of the proof of Theorem 7 . The algebraic reflexivity of the automorphism group can be proved in an easier way.

\section{Fréchet algebras of holomorphic functions.}

Consider now the full algebra $H(\Omega)$ equipped with the topology of uniform convergence on compact subsets of $\Omega$. In this case $H(\Omega)$, as a Fréchet space, is metrizable but there is no natural metric on $H(\Omega)$ and, therefore, there is no natural notion of isometry for $H(\Omega)$. We have, however, the following result about the automorphism group.

Theorem 8. Let $\Omega \subset \mathbb{C}$ be a simply connected domain. Then the automorphism group of $H(\Omega)$ is topologically reflexive if and only if $\Omega \neq \mathbb{C}$.

Proof. That the automorphism group of $H(\mathbb{C})$ is not topologically reflexive can be seen as follows: consider the automorphisms $\psi_{n}: H(\mathbb{C}) \longrightarrow$ $H(\mathbb{C})$ defined by

$$
\left(\psi_{n}(f)\right)(z)=f\left(\frac{z}{n}\right), \quad z \in \mathbb{C}, n \in \mathbb{N} .
$$

Let $\psi(f)=f(0)(f \in H(\mathbb{C}))$. Clearly, we have $\psi(f)=\lim _{n} \psi_{n}(f)$ for every $f \in H(\mathbb{C})$, but $\psi$ is not an automorphism of $H(\mathbb{C})$. This proves the "only if part".

For the converse, recall that every every automorphism of the algebra of all holomorphic functions on a domain is induced by a conformal selfmap of the underlying set [2], [3]. Just as above, we can assume that $\Omega=U$.

Let $\psi \in \operatorname{ref}_{t o}(\operatorname{Aut}(H(U)))$. As in the previous section, it is easy to see that any sequence of conformal mappings of $U$ onto itself has a subsequence 
which converges (uniformly on compact subsets of $U$ ) to either a map of the same kind or to a constant function of modulus 1 . One can readily check that this implies that for every $f \in A(\bar{U}) \subset H(U)$ we have $\psi(f) \in A(\bar{U})$ and the range of $\psi(f)$ is contained in that of $f$. Therefore, by GleasonKahane-Żelazko theorem $\psi$ is multiplicative on $A(\bar{U})$ and just as in the proof of Theorem 6 we infer that there is a function $\varphi: \bar{U} \rightarrow \bar{U}$ such that

$$
\psi(f)=f \circ \varphi, \quad f \in A(\bar{U})
$$

Considering $\psi(\mathrm{id})$ we find that $\varphi$ is either a conformal map of $U$ onto itself, or it is a constant function of modulus 1 . Since $\psi$ is supposed to be continuous and the polynomials are dense in $H(U)$, it follows that in the first case we have $\psi(f)=f \circ \varphi$ for every $f \in H(U)$, so in this case $\psi$ is an automorphism of $H(U)$. As for the second possibility, suppose that $\varphi \equiv \alpha$, where $\alpha$ is a constant of modulus 1 . Since the function $1 /(\alpha-\mathrm{id})$ is a element of $H(U)$, by the continuity of $\psi$ we obtain

$$
\psi\left(\frac{1}{\alpha-\mathrm{id}}\right)=\psi\left(\sum_{n} \frac{\mathrm{id}^{n}}{\alpha^{n+1}}\right)=\sum_{n} \frac{1}{\alpha} .
$$

This contradiction shows that the second possibility cannot occur. The proof is now complete.

Remark 8. Let $\Omega \subset \mathbb{C}$ be a bounded domain whose complement has finitely many components each of them with nonempty interior. We show that the automorphism group of $H(\Omega)$ is algebraically reflexive. Let $\psi$ : $H(\Omega) \longrightarrow H(\Omega)$ be a local automorphism. Since every automorphism of $H(\Omega)$ is induced by a conformal selfmap of $\Omega$ we see that $\psi$ can be considered as a local automorphism of $H^{\infty}(\Omega)$. By the second part of Remark 7, it follows that $\psi$ is an automorphism of $H^{\infty}(\Omega)$. Our topological condition on $\Omega$ was set to guarantee that, by Runge's approximation theorem $[26$, 13.9 Theorem], $H^{\infty}(\Omega)$ is dense in $H(\Omega)$. Therefore, by the continuity of $\psi$ we obtain that $\psi$ is a homomorphism of $H(\Omega)$. But the form of endomorphisms of $H(\Omega)$ is well-known. Using, for example, [3, Corollary], we have $\psi(f)=f \circ \varphi(f \in H(\Omega))$. Since $\varphi=\psi($ id $)$ is, by assumption, a conformal map, we obtain that $\psi$ is an automorphism of $H(\Omega)$.

Finally, we prove that the automorphism group of $H(\mathbb{C})$ is also algebraically reflexive. It is well-known that the conformal maps of $\mathbb{C}$ onto itself are precisely the affine functions $z \mapsto a z+b, a \neq 0$. Let $\psi: H(\mathbb{C}) \longrightarrow H(\mathbb{C})$ be a local automorphism. Assume that $\psi(\mathrm{id})=\mathrm{id}$. By the continuity of $\psi$, it is sufficient to prove that $\psi\left(\mathrm{id}^{n}\right)=\mathrm{id}^{n}$ holds for every $n \in \mathbb{N}$. Let 
$n \geq 3$. From the equality $\psi\left(\mathrm{id}+\mathrm{id}^{n}\right)=\psi(\mathrm{id})+\psi\left(\mathrm{id}^{n}\right)$ it follows that there are complex numbers $a, b, c, d$ with $a, c \neq 0$ such that

$$
(a z+b)+(a z+b)^{n}=z+(c z+d)^{n}, \quad z \in \mathbb{C} .
$$

Applying an appropriate affine transformation, the previous equality turns to

$$
z+z^{n}=\left(a^{\prime} z+b^{\prime}\right)+\left(c^{\prime} z+d^{\prime}\right)^{n},
$$

where $a^{\prime}, c^{\prime} \neq 0$. Comparing the coefficients of the polynomials above, since $1<n-1<n$, it follows that $d^{\prime}=0$ and then that $b^{\prime}=0$. We obtain $a^{\prime}=1,\left(c^{\prime}\right)^{n}=1$. It is not hard to see that this yields $\psi\left(\mathrm{id}^{n}\right)=\mathrm{id}^{n}$. It remains to check $\psi\left(\mathrm{id}^{2}\right)=\mathrm{id}^{2}$. Let $\psi\left(\mathrm{id}^{2}\right)=\varphi^{2}$, where $\varphi$ is an affine function. Picking any $n \geq 4$ and using a very similar argument as above but this time for $\psi\left(\mathrm{id}^{2}+\overline{\mathrm{id}}^{n}\right)=\psi\left(\mathrm{id}^{2}\right)+\psi\left(\mathrm{id}^{n}\right)$, we obtain

$$
\varphi=\frac{u_{2}}{u_{n}} \mathrm{id}
$$

where $u_{2}$ is a second, while $u_{n}$ is an $n$-th root of unity. Since this holds for every $n \geq 4$, we readily have $\varphi^{2}=\mathrm{id}^{2}$ and thus $\psi\left(\mathrm{id}^{2}\right)=\mathrm{id}^{2}$. This completes the proof of the algebraic reflexivity of $\operatorname{Aut}(H(\mathbb{C}))$.

To conclude sections 4, 5 and 6 , we remark that it would be interesting to extend our topological reflexivity results for more general domains on the plane as well as to give examples of exotic domains for which the corresponding spaces of holomorphic functions have nonreflexive isometry groups, or automorphism groups. It would be also interesting to treat similar problems for vector valued holomorphic functions instead of scalar valued ones (see [16], [17]).

\section{Spaces of continuous functions.}

In this section we give some examples concerning spaces of continuous functions. It is proved in [22, Theorem 2.2] that both $\operatorname{Aut}(C(K))$ and Iso $(C(K))$ are algebraically reflexive if $K$ is a first countable Hausdorff space. Our following example shows that reflexivity may fail even if $K$ lacks first countability at only one point. Observe that if $\Gamma$ is a discrete space, then for its one-point compactification $\Gamma \cup\{\infty\}$ we have $C(\Gamma \cup\{\infty\})=$ $c(\Gamma)=\mathbb{K} \oplus c_{0}(\Gamma)$. 
Example 2. Let $\Gamma$ be an uncountable index set. Then $\operatorname{Iso}(c(\Gamma))$ and $\operatorname{Aut}(c(\Gamma))$ are algebraically nonreflexive.

Proof. First, the construction in Example 1 gives us a non-surjective operator $T_{0}: c_{0}(\Gamma) \longrightarrow c_{0}(\Gamma)$ which is both a local surjective isometry and a local automorphism of $c_{0}(\Gamma)$. Since $c(\Gamma)=\mathbb{K} \oplus c_{0}(\Gamma)$, we can extend $T_{0}$ to $c(\Gamma)$ by putting

$$
T(\lambda \mathbf{1}+f)=\lambda \mathbf{1}+T_{0}(f), \quad \lambda \in \mathbb{K}, f \in c_{0}(\Gamma) .
$$

Clearly, $T$ is a non-surjective local automorphism and hence a local surjective isometry of $c(\Gamma)$. This completes the proof.

Our next example is a space of continuous functions whose set of local automorphisms is as large as it can be according to Remark 5. As usual, we denote by $\mathbb{N}^{*}$ the growth of $\mathbb{N}$ in its Stone-Cech compactification, that is, $\mathbb{N}^{*}=\beta \mathbb{N} \backslash \mathbb{N}$ which is a compact Hausdorff space.

Theorem 9. Every unital injective endomorphism of $C\left(\mathbb{N}^{*}\right)$ is a local automorphism. Therefore, the isometry group and the automorphism group of $C\left(\mathbb{N}^{*}\right)$ fail to be algebraically reflexive.

Proof. Let $\psi: C\left(\mathbb{N}^{*}\right) \longrightarrow C\left(\mathbb{N}^{*}\right)$ be a unital injective endomorphism. As it is well-known, every unital endomorphism of a $C(K)$ space is induced by a continuous selfmap of $K$. Hence there is a surjective continuous mapping $\varphi: \mathbb{N}^{*} \longrightarrow \mathbb{N}^{*}$ for which $\psi(f)=f \circ \varphi$ for all $f \in C\left(\mathbb{N}^{*}\right)$. Since $f$ and $\psi f$ have the same range, there is an homeomorphism $\varphi_{f}$ of $\mathbb{N}^{*}$ such that $\psi(f)=f \circ \varphi_{f}$ as a consequence of the following fact about the structure of $\mathbb{N}^{*}$ [28, p. 83]: if $K$ is a compact space of topological weight at most $\aleph_{1}$ and $f$ and $g$ are continuous maps from $\mathbb{N}^{*}$ onto $K$, then there is a homeomorphism $\phi$ of $\mathbb{N}^{*}$ such that $g=f \circ \phi$. Clearly, this yields that $\psi$ is both a local automorphism and a local surjective isometry, although $\psi$ is never surjective unless $\varphi$ is injective. Since $\mathbb{N}^{*}$ contains two disjoint copies of itself which are clopen in $\mathbb{N}^{*}$ (see $\left.[28,3.10,3.14,3.15]\right)$, the existence of a surjective non-injective mapping $\varphi: \mathbb{N}^{*} \longrightarrow \mathbb{N}^{*}$ is obvious. This completes the proof.

Our final examples show that there is no much relation between the reflexivity of the isometry group of $X$ and that of its dual $X^{*}$. This solves in part Problem 2 at the end of [22].

Example 3. a) There is a Banach space $X$ such that $\operatorname{Iso}(X)$ is topologically reflexive but Iso $\left(X^{*}\right)$ fails to be algebraically reflexive. 
b) There is a Banach space $Y$ with algebraically nonreflexive isometry group such that $\operatorname{Iso}\left(Y^{*}\right)$ is algebraically reflexive.

Proof. We first prove b). Let $Y=l_{1}(\Gamma)$, where $\Gamma$ is an uncountable index set. By Example 1, Iso $(Y)$ is algebraically nonreflexive. However, the isometry group of $Y^{*}=l_{\infty}(\Gamma)$ is algebraically reflexive; this can be proved, mutatis mutandis, as in the countable case (see [1, Proposition 6]).

To verify a), let $K$ be Cook's continuum having no nontrivial continuous surjection onto itself [4]. We show that $\operatorname{Iso}(C(K))$ is topogically reflexive. Let $T \in \operatorname{ref}_{t o}(\operatorname{Iso}(C(K)))$. By Theorem 5, one has

$$
T f=\tau(f \circ \varphi)
$$

where $\tau$ is unimodular and $\varphi$ is a continuous surjection of $K$ onto itself. Hence $\varphi$ is the identity on $K$ and we obtain that $T$ is surjective. On the other hand, by general representation results, one has

$$
C(K)^{*}=l_{1}(K) \oplus_{1} L_{1}(\mu)
$$

where $\mu$ is a non-atomic measure. Since $K$ is uncountable (no continuum is countable), it follows from Theorem 3 that the isometry group of $C(K)^{*}$ is algebraically nonreflexive.

We close the paper with the following open problem. Let $X$ be a locally compact Hausdorff space and denote by $C_{0}(X)$ the Banach algebra of all continuous real or complex valued functions on $X$ which vanish at infinity. If $C_{0}(X)$ is separable (this means that the one-point compactification of $X$ is metrizable), does it follow that $\operatorname{Iso}\left(C_{0}(X)\right)$ is algebraically reflexive? If this was the case, we would get that the isometry group of any separable commutative $C^{*}$-algebra is algebraically reflexive and we would have some hope to obtain positive reflexivity results for the isometry group of some classes of separable $C^{*}$-algebras.

\section{References.}

[1] Batty, C. J. K., Molnár, L., On topological reflexivity of the groups of *-automorphisms and surjective isometries of $B(H)$. Arch. Math. 67 (1996), 415-421.

[2] Bers, L., On rings of analytic functions. Bull. Amer. Math. Soc. 54 (1948), 311-315.

[3] Burckel, R. B., SAeki, S., Additive mappings on rings of holomorphic functions. Proc. Amer. Math. Soc. 89 (1983), 79-85. 
[4] Cook, H., Continua which admit only the identity mapping onto nondegenerate subcontinua. Fundam. Math. 60 (1967), 241-249.

[5] Duren, P. L., Theory of $H^{p}$ spaces. Academic Press, 1970.

[6] Forelli, F., The isometries of $H^{p}$. Canad. J. Math. 18 (1964), 721-728.

[7] Gordon, Y., Lewis, D. R., Isometries of diagonally symmetric Banach spaces. Israel J. Math. 28 (1977), 45-67.

[8] Greim, P., Jamison, J. E., Kaminska, A., Almost transitivity of some function spaces. Math. Proc. Camb. Phil. Soc. 116 (1994), 475-488.

[9] Jarosz, K., Any Banach space has an equivalent norm with trivial isometries. Israel J. Math. 64 (1988), 49-56.

[10] Kadison, R. V., Local derivations. J. Algebra 130 (1990), 494-509.

[11] KaKUtani, S., Rings of analytic functions. Lectures on functions of a complex variable, Ann Arbor, 1955.

[12] Kalton, N. J., Peck, N. T., Roberts, J. W., An F-space sampler. Lecture Notes in Math. 89 London Math. Soc., 1984.

[13] Lamperti, J., The isometries of certain function spaces. Pacific J. Math. 8 (1958), 459-466.

[14] LARSOn, D. R., Reflexivity, algebraic reflexivity and linear interpolation. Amer. J. Math. 110 (1988), 283-299.

[15] DeLeeuw, K., Rudin, W., Wermer, J., The isometries of some function spaces. Proc. Amer. Math. Soc. 11 (1960), 694-698.

[16] Lin, P.-K., The isometries of $H^{\infty}($ E) Pacific J. Math. 143 (1990), 69-77.

[17] Lin, P.-K., The isometries of $H^{p}(K)$ J. Austral. Math. Soc. 50 (1991), 23-33.

[18] Lindenstrauss, J., Tzafriri, L., Classical Banach Spaces I. Springer, 1977.

[19] Molnár, L., The set of automorphisms of $B(H)$ is topologically reflexive in $B(B(H))$. Studia Math. 122 (1997), 183-193.

[20] Molnár, L., Reflexivity of the automorphism and isometry groups of $C^{*}$-algebras in BDF theory. Arch. Math. 74 (2000), 120-128.

[21] Molnár, L., Győry, M., Reflexivity of the automorphism and isometry groups of the suspension of $B(H)$. J. Funct. Anal. 159 (1998), 568-586.

[22] Molnár, L., Zalar, B., Reflexivity of the group of surjective isometries of some Banach spaces. Proc. Edin. Math. Soc. 42 (1999), 17-36.

[23] Molnár, L., Zalar, B., On local automorphisms of group algebras of compact groups. Proc. Amer. Math. Soc. 128 (2000), 125-134.

[24] Palmer, T. W., Banach Algebras and The General Theory of *-Algebras. Vol. I. Encyclopedia Math. Appl. 49 Cambridge University Press, 1994.

[25] Rolewicz, S., Metric Linear Spaces. Monografie Matematyczne 56 PNW and D. Reidel, 1984. 
[26] Rudin, W., Real and Complex Analysis. Tata McGraw-Hill Publishing Co. Ltd., 1983.

[27] Stern, J., Le groupe des isometries d'un espace de Banach. Studia Math. 64 (1979), 139-149.

[28] Walker, R. C., The Stone-Čech compactification. Ergebnisse der Math. und ihrer Grenzgebiete 83 Springer, 1974.

Recibido: 8 de noviembre de 2000

Félix Cabello Sánchez*

Departamento de Matemáticas

Universidad de Extremadura Avenida de Elvas, 06071-Badajoz, SPAIN

fcabello@unex.es

and

Lajos Molnár ${ }^{\dagger}$

Institute of Mathematics and Informatics Lajos Kossuth University H-4010 Debrecen, P.O. Box 12, HUNGARY molnarl@math.klte.hu

* Supported in part by DGICYT project PB97-0377 and HI project 19970016.

$\dagger$ Supported by the Hungarian National Foundation for Scientific Research (OTKA) and by the Ministry of Education (FKFP). 